\title{
Severe Liver Fibrosis and Association with Plasma Inflammatory Biomarkers among HIV/HCV Co- infected Patients in China :a Cross-Sectional Study
}

\section{Xiaochen Chen}

Fudan University School of Public Health

Xing Liu

Fudan University School of Public Health

Song Duan

Dehong Prefecture Center for Disease Control and Prevention, Mangshi

Renhai Tang

Dehong Prefecture Center for Disease Control and Prevention, Mangshi

Sujuan Zhou

Fudan University School of Public Health

Runhua Ye

Dehong Prefecture Center for Disease Control and Prevention, Mangshi

Yuecheng Yang

Dehong Prefecture Center for Disease Control and Prevention

Jibao Wang

Dehong Prefecture Center for Disease Control and Prevention, Mangshi

Shitang Yao

Dehong Prefecture Center for Disease Control and Prevention, Mangshi

\section{Meiyang Gao}

Fudan University School of Public Health

\section{Yingying Ding}

Fudan University School of Public Health

$\mathrm{NaHe}$ ( $\square$ nhe@shmu.edu.cn )

Fudan University School of Public Health

\section{Research article}

Keywords: HIV/HCV co-infected; microbial translocation; liver fibrosis; inflammation

Posted Date: October 10th, 2019

DOI: https://doi.org/10.21203/rs.2.15814/v1 
License: (c) (i) This work is licensed under a Creative Commons Attribution 4.0 International License. Read Full License 


\section{Abstract}

Background: Immune dysregulation among HIV/HCV co-infected patients with impaired liver function is common. Thus, this study aimed to evaluate the association of liver fibrosis with microbial translocation and related inflammation among HIV/HCV co-infected patients.

Methods: This cross-sectional study involved $343 \mathrm{HIV} / \mathrm{HCV}$ co-infected patients who received cART. All patients had current blood biochemical testing data. We measured SCD14 and 27 serum cytokines concentrations using the Hycult Biotech sCD14 ELISA kit and Bio-plex Human Cytokine 27-plex Assay, respectively. We compared the concentrations of each marker between severe liver fibrosis and mild liver fibrosis. Odds ratios (ORs) and $95 \%$ confidence intervals $(95 \% \mathrm{Cls})$ for the association of each marker with severe liver fibrosis were estimated using logistic regression.

Results: Of the 343 HIV/HCV coinfect-ed patients enrolled, 188 (54.8\%) had severe liver fibrosis (FIB-4 $>3.25)$. Patients with higher FIB-4 score (>3.25vs. $\leq 3.25$ ) had higher plasma level of IL-1 $\beta$, IL-6, IL-7, IL-9, IL-12, IL-15, IL-17, GM-CSF, IFN-y, TNF-a, IL-4, IL-10, IL-13, BasicFGF and MCP-1. Multivariate logistic regression analysis showed that increased plasma level of IL-1 $\beta$, IL-6, IL-7, IL-12, IL-17, GM- CSF, IFN-y, IL4, IL -10, MCP-1, Eotaxin, BasicFGF and SCD14 were linked to severe liver fibrosis in our study.

Conclusions: Severe liver fibrosis are associated with increased microbial translocation plasma inflammatory biomarkers among HIV/HCV co-infected patients.

\section{Background}

Human immunodeficiency virus (HIV) and Hepatitis C virus (HCV) co-infection is a worldwide serious public health concern because of increased mortality and disease burden[1, 2]. Compared to HCV monoinfected patients, HIV/HCV co-infected patients have more frequent and accelerated progression to fibrosis, cirrhosis and end-stage liver disease (ESLD)[3]. Differ from western countries, direct-acting antivirals (DAAs) even peg-interferon plus ribavirin (PR) in China especially rural area cannot be widely used because of the high price[32].So the burden of disease caused by HIV/HCV may be heavier in China. Pathogenesis of progressive liver fibrosis in HIV/HCV co-infected is complex and multifactorial [4], and is mainly related to persistent HCV infection with altered cellular immunity as well as immune activation with elevated pro-inflammatory and profibrogenic cytokines[5]. More specifically, HIV infection induces immune suppression and leads to CD4 T-cell depletion, causes persistent innate and acquired immune activation, and directly stimulates Kupffer cells and hepatic stellate cells (HSCs) to secrete either profibrotic cytokines or type 1 collagen through a C-C chemokine receptor-5 (CCR5)-dependent pathway[5]. In addition, the persistence of HCV infection due to weakened CD4 and CD8T cell responses caused by HIV[6] contributes to increased rates of liver fibrosis[7-9].

Moreover, microbial translocation (MT), which is thought to be associated with loss of mucosal barrier function and increased intestinal permeability secondary to immune dysregulation and/or alterations in the intestinal microbiome, has often been observed among HIV-infected individuals[10]. Blood microbial 
components including peptidoglycan, lipoteichoic acid, lipopolysaccharide (LPS) and flagellin may directly promote liver fibrosis in HIV/HCV co-infected patients by stimulation of HSCs and/or Kupffer cells with LPS binding protein (LBP) and soluble CD14 (sCD14). These microbial components may enhance local hepatic inflammatory immune responses and activation-induced liver cell death[4].

Thus, the above-mentioned mechanisms jointly emphasize the important role played by inflammation in liver fibrosis among HIV/HCV co-infected patients. Cirrhosis-associated dysregulation of immune responses is reflected by increased production and elevated serum levels of pro-inflammatory cytokines and upregulated expression of cell activation markers[11]. Kupffer cells generate IL-1, IL-6, IL12, IL-18 and also release anti-inflammatory cytokines, including IL-10 after stimulation by LPS[12]. Upregulation of IL-4, IL13, TGF-1 and platelet-derived growth factor (PDGF) was observed during fibrogenesis, while among HIV-infected patients, gp120 may induce HSC accumulation by secretion of monocyte chemoattractant protein-1 (MCP-1) by HSCs[13]. Furthermore, HIV suppression by combination antiretroviral therapy (cART) among HIV/HCV co-infected patients may decrease inflammation and immune activation and slows down the progression of liver disease [14], whereas the levels of plasma inflammatory biomarkers remain abnormal in many individuals[15]. However, the inflammatory profile among HIV/HCV co-infected patients with different levels of liver fibrosis has not been well established. Up to now, studies of the role of microbial translocation and plasma inflammatory biomarkers in liver fibrosis are limited by small sample sizes and small number of examined biomarkers[16-18]. To fill this gap, the present study aimed primarily to examine the association of liver fibrosis with microbial translocation and related inflammation among HIV/HCV co-infected patients in the era of cART.

\section{Methods}

\section{Study sample}

The present cross-sectional study was conducted in Dehong Prefecture of Yunnan Province at China's southwest border, where the first China's indigenous HIV outbreak was reported in 146 infected heroin users in 1989, and injection drug use (IDU) had been the predominant mode of HIV transmission through the early 2000s and continues to be an important source of HIV infection[19, 20]. By March 2016, 1017 HIV/HCV co-infected patients with 170 (16.7\%) deaths had been reported and registered in the Comprehensive Response Information Management System (CRIMS) for HIV/AIDS in China, which is a unified web-based national information system with the capacity of longitudinal assessment and monitoring of HIV-infected patients' health status[21]. Out of the remaining 847 patients, 463 (54.7\%) were identifiable during the study period from April to October in 2016. Of them, 390 (84.2\%) were receiving cART and gave informed consent to participate in the present study. Forty-seven patients were further excluded from the study due to missing data on biochemical measures about aspartate aminotransferase (AST), alanine aminotransferase (ALT), and platelet count (PLT) that are necessary to evaluate liver fibrosis status. Thus, a total of $343 \mathrm{HIV} / \mathrm{HCV}$ co-infected patients were included in the final analysis. These 343 participants had no significant differences with the 120 identifiable yet excluded 
$\mathrm{HIV/HCV}$ co-infected patients in the distribution of age, sex, marital status, HIV transmission route, HBsAg serostatus, baseline CD 4 cells count, years on cART, current HCV RNA and HIV RNA levels except for ethnicity (Table S1).

\section{Data Extraction}

Demographical and clinical epidemiological data were extracted from the CRIMS. The data included age, sex, marital status, ethnicity, HIV transmission route, date of CART initiation, antiretroviral regimen, and CD4 cell counts at CART initiation and follow-up visits.

\section{Blood testing}

\section{Biochemical tests for liver fibrosis assessment}

Biochemical measurements of AST, ALT and PLT were performed using an automatic biochemistry analyzer (Beckman Coultner, USA), according to the manufacturer's protocol. Liver fibrosis was assessed by FIB-4 score, using Sterling's formula calculated as (AST [IU/L] $\times$ age [years]) / (PLT [10\%/L] × $\left.\operatorname{ALT}[I U / L]^{1 / 2}\right)[22]$. This is an international recognized well-established noninvasive indicator of liver fibrosis in HIV/HCV co-infected patients[23]. FIB-4 is generally divided into three categories: no or mild liver fibrosis with FIB-4 $<1.45$, intermediate liver fibrosis with $1.45 \leq \mathrm{FIB}-4 \leq 3.25$, and severe liver Fibrosis with FIB-4 $>3.25$. In this study, we were more concerned with severe liver fibrosis (FIB-4 >3.25) [24].

\section{HCV RNA Quantification and genotyping}

Plasma HCV viral RNA was extracted (Roche diagnostic products (Shanghai) Co., Ltd., China) and quantified by a real-time polymerase chain reaction (RT-PCR) technique using commercially available kits for the quantification of HCV RNA (PCR-Fluorescent Probing, PG Biotech Ltd., Shenzhen, China). The limit of detection was 500 copies $/ \mathrm{ml}$, and the linear range of HCV RNA quantification was from $1.0 \times 10^{3}$ to $5.0 \times 10^{7}$ copies $/ \mathrm{ml}$.

Amplification was completed by a nested PCR with E1- or NS5B-specific primers. Splicing, proofreading, and aligning sample sequences were performed using ChromasPro 1.5 and BioEdit7.0.9.0 software. HCV genotype reference sequences were retrieved from the HCV database (http://hcv.lanl.gov/content/sequence/HCV/ToolsOutline.html). The phylogenetic tree was established by the Neighbor-joining method of MEGA 7.0 software. The Bootstrap repeat detection value was set to 1000 times, and the HCV gene subtype was further determined according to the phylogenetic tree[25]. 


\section{Multiplex cytokine bead assay and ELISA}

Twenty-seven cytokines, chemokines and growth factors cytokines in plasma specimens were quantified by a multiplex analysis performed on the BioPlex ${ }^{\circledR} 200$ Multiplex System platform using the Bio-Plex Human 27-plex panel of cytokines/chemokines/growth factors (Bio-Rad, Hercules, CA, USA), according to the manufacturer's instructions. These biomarkers included 1) proinflammatory biomarkers such as interleukin (IL) $-1 \beta, I L-2, I L-6, I L-7, I L-8, I L-9, I L-12, I L-15, I L-17$, granulocyte colony stimulating factor (G-CSF), granulocyte macrophage colony stimulating factor (GM-CSF), Interferon-y (IFN- - ), and tumor necrosis factor (TNF-a); 2) anti-inflammatory biomarkers such as IL $-1 \mathrm{ra}, \mathrm{IL}-4, \mathrm{IL}-5, \mathrm{IL}-10$, and IL-13; 3) chemokines such as IFN- - -inducible protein (IP-10), monocyte chemoattractant protein-1 (MCP-1), macrophage inflammatory proteins-1alpha (MIP-1a), MIP-1b, and regulated upon activation normal T-cell expressed and secreted (RANTES); and 4) growth factors such as Eotaxin, fibroblast growth factor 2 (Basic FGF), platelet-derived growth factor (PDGF-BB), and vascular endothelial growth factor (VEGF). The detection limit for each molecule was determined by the recovery of the corresponding standard, and the lowest values with more than $70 \%$ recovery were set as the lowest detection limits. Measurements less than the lower limit of quantification (LLOQ) were assigned a value of half the LLOQ, and measurements more than the upper limit of quantification (ULOQ) were assigned a value of twice the ULOQ for each marker.

Plasma SCD14, as an indirect surrogate marker of microbial translocation (MT), was measured according to the manufacturer's protocol for commercial enzyme-linked immunosorbent assay (ELISA) (Hycult Biotech, Wayne PA, USA).

\section{Other tests}

Human hepatitis B virus surface antigen (HBsAg) was tested by ELISA technique (Wan Tai Biomedical Co. Ltd, Beijing, China). CD4 cell counts were assessed by FACSCount (Becton, Dickinson and Co., San Jose, CA, USA).

\section{Statistical analysis}

Group comparisons were assessed using chi-square test or Fisher's exact test for categorical variables and t-test or Mann-Whitney $U$ test for continuous variable. Log10-transformation were conducted for variables of plasma cytokines levels for further statistical and regression analyses. A multiple logistic regression analysis with adjustment for age, sex, ethnicity, current HIV RNA, current HCV RNA, current CD4 cell counts, years since cART and ART regimen type was undertaken to explore the correlation of liver fibrosis with the plasma level of each of the twenty-seven cytokines and SCD14. The odd ratio (OR) and $95 \%$ confidence interval $(95 \% \mathrm{Cl})$ represent the risk of liver fibrosis per one log-unit change in plasma cytokine concentration. Spearman correlations were computed to explore associations between the 
plasma inflammatory biomarkers. Statistical significance was defined as $p<0.05$ and Bonferroni $p$ $<0.002(0.05 / 27 \approx 0.002)$ for multiple comparison adjustment [26]. All statistical analyses were performed using R software (version 3.3.2).

\section{Results}

\section{Demographical and clinical characteristics}

The median (IQR) age at baseline was 35.4 (31.3-39.3) years old. The majority of the participants were male, injection drug users (IDUs), seronegative for hepatitis B surface antigen (HBsAg), detectable for plasma HCV RNA but undetectable for plasma HIV RNA. Around half of the participants were non-Han ethnicities, i.e., ethnic minorities (54.2\%), currently married (51.9\%), and of HCV subtype 3 (47\%) ( Table 1).

\section{Prevalence of Severe Liver fibrosis}

Among all participants, 75 (21.9\%) had no or mild liver fibrosis, 80 (23.3\%) had intermediate liver fibrosis, and $188(54.8 \%)$ had severe liver fibrosis. Compared to patients without severe liver fibrosis, those with severe liver fibrosis were significantly different in age, education, cART regimen, and years on cART(Table 1).

\section{Plasma levels of inflammatory biomarkers}

Participants with sever liver fibrosis (FIB-4 3.25) had significantly higher plasma levels of IL-1 $\beta$, IL-4, IL-6, IL-7, IL-9, IL-10, IL-12, IL-13, IL-15, IL-17, IFN- $\gamma$, TNF-a, MCP-1, GM-CSF, Basic FGF and VEGF

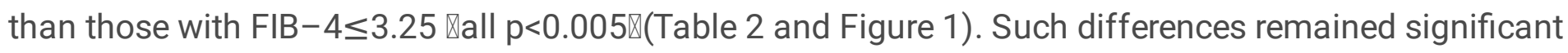
for IL-6, IL-10, IFN-y, GM-CSF and Basic FGF after Bonferroni correction for multiple comparisons. No significant difference in SCD14 was observed between the two groups.

\section{Correlations of inflammatory biomarkers and their associations with severe liver fibrosis}

Most of the 27 cytokines were significantly correlated with each other with the exception of RANTES, which was not correlated with most of the other cytokines (Figure 2). The correlation coefficients are shown as a supplement in Table S2.

Given the high correlation between the 27 cytokines, separate multiple logistic regression models adjusting for potential confounders were performed to examine the association of sever liver fibrosis with 
the each of the 27 cytokines and SCD14 levels. Severe liver fibrosis was shown to be associated with higher levels of eleven out of the twenty-seven cytokines with a significance level of 0.05 , only Basic FGF was found to be positively and significantly associated with severe liver fibrosis after Bonferroni correction for multiple comparisons $(\mathrm{aOR}=1.82 ; 95 \% \mathrm{Cl}: 1.26-2.66 ; \mathrm{p}=0.002)$. Furthermore, plasma sCD14 was significantly associated with severe liver fibrosis $(a O R=1.14 ; 95 \% \mathrm{Cl}: 1.01-1.30 ; p=0.048$. Figure 3).

\section{Discussion}

In the present study, $54.8 \%$ of HIV/HCV co-infected patients had severe liver fibrosis or cirrhosis. The prevalence was higher than studies conducted in western countries which range from $22.9 \%$ to $40.3 \%$ [27-30]. Among one study conducted in Southeast Asia including $380 \mathrm{HIV} / \mathrm{HCV}$ co-infected patients, $40.3 \%$ had liver fibrosis or cirrhosis[31]. The discrepancy is possibly attributed to the fact that these patients lack of antiviral therapy for viral hepatitis $\mathrm{C}$.

HIV and HCV infection are both characterized by systemic inflammation with increased levels of blood inflammatory cytokines. HIV infection may alter the pattern of cytokine release and exacerbate immunemediated inflammatory responses[33]. Although plasma inflammatory markers tend to decrease in $\mathrm{HIV} / \mathrm{HCV}$ co-infected patients after CART, they still remain much higher than healthy controls [6, 34]. In the present study, we found that HIV/HCV co-infected patients with severe liver fibrosis were living with an elevated level of inflammatory cytokines including pro-inflammatory cytokines, anti-inflammatory cytokines, chemoattractant cytokines, growth factors and the microbial translocation surrogate marker sCD14. This indicates that progressive and advanced liver fibrosis in HIV/HCV co-infected is mainly an inflammatory syndrome $[6,35]$.

Many studies have shown that inflammation plays an important role in the development of liver fibrosis among HIV/HCV co-infected patients[36, 37]. In this regard, levels of cytokines, chemokines and growth factors closely related to inflammation were altered. We found that patients with cirrhosis who had FIB-4 $>3.25$ showed a significantly higher plasma level of various inflammatory makers (IL-1 $13, \mathrm{IL}-6, \mathrm{IL}-7$, IL9, IL-12, IL-15, IL-17, GM-CSF, IFN- $y$, TNF-a, IL-4, IL-10, IL-13, BasicFGF and MCP-1). Although the median plasma level of SCD14 was higher in the FIB-4 >3.25 group than FIB-4 $\leq 3.25$, there was no significant difference.

Chronic inflammation and fibrosis are inextricably linked, and the interactions between immune effector cells, local fibroblasts and tissue macrophages at sites of scar formation determine the outcome of liver injury. With improved understanding of the processes that govern inflammation and fibrosis, it has become clear that both the adaptive and innate immune systems are involved in the regulation of fibrosis[38].

In our study, multivariate logistic regression analysis shows that increased plasma levels of IL-1 $1 \beta$, IL -6 , $\mathrm{IL}-7$, IL-12, IL-17, GM- CSF, IFN-ץ, IL-4, IL -10, MCP-1, Eotaxin, BasicFGF and sCD14 are linked to severe liver fibrosis. Our findings are in concordance with previous studies that show plasma levels of 
proinflammatory, including TNF-a, IL-6, IL-8, IL-12, are profibrogenic and induce liver damage[39, 40]. In addition, these studies show that cirrhotic patients display increased production of IFN-y, IL-4 and IL-13 [41]. However, the role of IFN- $\gamma$ in liver fibrosis is controversial[42]. IFN- $\gamma$ in these studies show that IFN- $\gamma$ is significantly negatively correlated with liver fibrosis[39] [43], but IFN-y also proves to have proinflammatory effects that can aggravate disease progression and organ dysfunction[44]. Our study shows a positive correlation between IFN- $\gamma$ levels and liver fibrosis. The association between IL-17 and fibrosis is also controversial. Sheila et al found an inverse correlation between IL -17 and aminotransferase-to-platelet ratio index (APRI) [45]; to the contrary, Meng et al found that IL-17 could exacerbate liver fibrosis[46]. In our study, we found that increased IL-17 concentration is associated with higher FIB-4 score. Regarding IL-2, a previous study found a negative correlation of IL-2 with cirrhosis[47]. However, our data shows that IL-2 levels increase as fibrosis increases.

Anti-inflammatory cytokines, including IL $-4 \mathbb{Q} \mathrm{IL}-10$ and $\mathrm{IL}-13$, are profibrogenic cytokines, and their levels increase in coinfected patients [48,49], which is in accordance with our findings.

Chemokines may promote inflammation through the recruitment of lymphocytes to the liver parenchyma in chronic hepatitis $\mathrm{C}$ virus (HCV) infection [50]. MIP-1a is a profibrogenic chemokine. Tazi, et al found that cirrhotic monocytes spontaneously produce chemokines (MCP-1) unlike normal monocytes[51]. Although increased MIP-1a increased the risk of developing cirrhosis in our study, it was not statistically significant. Many studies have shown that plasma IP-10 levels increased as liver fibrosis increased in HCV or HIV / HCV co-infected [33, 52, 53, 54], but our data did not find this association.

As for now, studies on the involvement of growth factors other than TGF- $\beta 1$ in liver fibrosis are rare. In our study, multivariate logistic models indicate that increased BasicFGF and Eotaxin levels persist as predictors for liver cirrhosis.

sCD14 is a significant biomarker related to microbial translocation since it reflects the host's response to microbial translocation [55]. Considering the limitations of the limulus test used to detect LPS, it is technically difficult to measure, and the results are often inconsistent [56]. Instead we can use SCD14 as an indirect biomarker of microbial translocation. Our study shows that increased SCD14 increases the risk of severe liver fibrosis, consistent with the findings of French et al[57].

\section{Limitations}

This study has several limitations. Firstly, due to cross-sectional design, this study fails to establish the direction of causality, e.g., inflammation may be a cause or response to liver cirrhosis. Secondly, we did not include factors known to influence the risk of liver fibrosis that could alter levels of immune/inflammatory markers such as alcohol abuse. Thirdly, we did not set a control group of HCVmonoinfected or HIV-monoinfected patients, and we are not able to know information of possible differential biomarkers from them. 


\section{Conclusions}

In summary, severe liver fibrosis are associated with increased microbial translocation plasma inflammatory biomarkers among HIV/HCV co-infected patients. Our findings provide preliminary evidence that immune/inflammatory markers may be useful predictors of liver fibrosis. Further research using longitudinal design is warranted to determine the causal relationship between liver fibrosis and inflammation, in order to look for the optimal care and management of HIV/HCV co-infected patients in China.

\section{Abbreviations}

HIV:Human immunodeficiency virus;HCV: Hepatitis C virus; ORs: Odds ratios; 95\%Cls: 95\% confidence intervals; ESLD:end-stage liver disease; HSCs:hepatic stellate cells; CCR5: C-C chemokine receptor-5; LPS: lipopolysaccharide; LBP: LPS binding protein; sCD14: soluble CD14; CART: combination antiretroviral therapy; IDU: injection drug use; CRIMS: Comprehensive Response Information Management System; AST: aspartate aminotransferase; ALT: alanine aminotransferase; PLT: platelet count; IRB: Institutional Review Board; FIB-4:fibrosis index-4; MT: microbial translocation;

\section{Declarations}

\section{Acknowledgements}

We would like to thank all participants of the study. We thank Emily Kestenbaum from Medical School for International Health at Ben Gurion University for editorial assistance.

\section{Author's contributions:}

The study was conceived and supervised by HN.TRH, ZSJ, YRH, YYC, WJB, YST, GMY and DYY conducted the survey. CXC analyzed the data and drafted the manuscript. LX and HN critically reviewed and revised the manuscript. All authors commented on drafts and read an approved the final manuscript.

\section{Funding}

This work was supported by the Natural Science Foundation of China (Grant No. 81773485; 81373062), the National Science and Technology Major Projects on Infectious Diseases of China (Grant No. 2018ZX10721102-004), and Shanghai Municipal Health Commission (Grant No. GWTD2015S05).

\section{Availability of data and materials}

The datasets used during the study are available from the corresponding author and provided on a reasonable request. 
The study was approved by the Institutional Review Board (IRB) of Fudan University, Shanghai, China. Written informed consent was obtained from all study participants.

Consent for publication

Not applicable

Competing interests

The authors declare that they have no competing interests.

Authors details

${ }^{1}$ Department of Epidemiology, School of Public Health, and the Key Laboratory of Public Health Safety of Ministry of Education, Fudan University, Shanghai, China, Na He, Xiaochen Chen, Xing Liu, Sujuan Zhou, Meiyang Gao, Yingying Ding, PhD;

${ }^{2}$ Key Laboratory of Health Technology Assessment of Ministry of Health, Fudan University, Shanghai, China, $\mathrm{Na} \mathrm{He}$, Xiaochen Chen;

${ }^{3}$ Dehong Prefecture Center for Disease Control and Prevention, Mangshi, Yunnan Province, China, Song Duan, Renhai Tang, Runhua Ye, Yuecheng Yang, Jibao Wang, Shitang Yao.

\section{References}

1.Joshi D, O'Grady J, Dieterich D, Gazzard B, Agarwal K: Increasing burden of liver disease in patients with HIV infection. Lancet (London, England) 2011, 377(9772):1198-1209.

2.Platt L, Easterbrook P, Gower E, McDonald B, Sabin K, McGowan C, Yanny I, Razavi H, Vickerman P: Prevalence and burden of HCV co-infection in people living with HIV: a global systematic review and meta-analysis. The Lancet Infectious diseases 2016, 16(7):797-808.

3.Lo Re V, 3rd, Kallan MJ, Tate JP, Localio AR, Lim JK, Goetz MB, Klein MB, Rimland D, RodriguezBarradas MC, Butt AA et al: Hepatic decompensation in antiretroviral-treated patients co-infected with HIV and hepatitis $C$ virus compared with hepatitis $C$ virus-monoinfected patients: a cohort study. Annals of internal medicine 2014, 160(6):369-379.

4.Page EE, Nelson M, Kelleher P: HIV and hepatitis C coinfection: pathogenesis and microbial translocation. Current opinion in HIV and AIDS 2011, 6(6):472-477.

5.Bruno R, Galastri S, Sacchi P, Cima S, Caligiuri A, DeFranco R, Milani S, Gessani S, Fantuzzi L, Liotta F et al: gp120 modulates the biology of human hepatic stellate cells: a link between HIV infection and liver fibrogenesis. Gut 2010, 59(4):513-520. 
6.Bruno R, Sacchi P, Puoti M, Maiocchi L, Patruno SF, Cima S, Filice G: Pathogenesis of liver damage in HCV-HIV patients. AIDS reviews 2008, 10(1):15-24.

7.Rotman Y, Liang TJ: Coinfection with hepatitis C virus and human immunodeficiency virus: virological, immunological, and clinical outcomes. Journal of virology 2009, 83(15):7366-7374.

8.Operskalski EA, Kovacs A: HIV/HCV co-infection: pathogenesis, clinical complications, treatment, and new therapeutic technologies. Current HIV/AIDS reports 2011, 8(1):12-22.

9.Kim AY, Chung RT: Coinfection with HIV-1 and HCV-a one-two punch. Gastroenterology 2009, 137(3):795-814.

10.Dinh DM, Volpe GE, Duffalo C, Bhalchandra S, Tai AK, Kane AV, Wanke CA, Ward HD: Intestinal microbiota, microbial translocation, and systemic inflammation in chronic HIV infection. The Journal of infectious diseases 2015, 211(1):19-27.

11.Albillos A, Lario M, Alvarez-Mon M: Cirrhosis-associated immune dysfunction: distinctive features and clinical relevance. Journal of hepatology 2014, 61(6):1385-1396.

12.Nakamoto N, Kanai TJFii: Role of toll-like receptors in immune activation and tolerance in the liver. 2014, 5:221.

13.Sacchi P, Cima S, Zuccaro V, Columpsi P, Sarda C, Mariani M, Puoti M, Bruno RJAr: Understanding the mechanisms of fibrogenesis in HIV/HCV-coinfected patients: implications for clinical practice. 2015, 17(3):159-170.

14.Sitia G, De Bona A, Bagaglio S, Galli L, Paties CT, Uberti-Foppa C, Guidotti LG, Lazzarin A, Morsica G: Naive HIV/HCV-coinfected patients have higher intrahepatic pro-inflammatory cytokines than coinfected patients treated with antiretroviral therapy. Antiviral therapy 2006, 11(3):385-389.

15.Hunt PW, Lee SA, Siedner MJ: Immunologic Biomarkers, Morbidity, and Mortality in Treated HIV Infection. The Journal of infectious diseases 2016, 214 Suppl 2:S44-50.

16.Peters L, Neuhaus J, Duprez D, Neaton JD, Tracy R, Klein MB, Mocroft A, Rockstroh J, Dore G, Lundgren JDJJoCV: Biomarkers of inflammation, coagulation and microbial translocation in HIV/HCV co-infected patients in the SMART study. 2014, 60(3):295-300.

17.Shmagel KV, Saidakova EV, Shmagel NG, Korolevskaya LB, Chereshnev VA, Robinson J, Grivel JC, Douek DC, Margolis L, Anthony DDJHm: Systemic inflammation and liver damage in HIV/hepatitis C virus coinfection. 2016, 17(8):581-589.

18.Hu S, Ghabril M, Amet T, Hu N, Byrd D, Yang K, Vuppalanchi R, Saxena R, Desai M, Lan JJPo: HIV-1 coinfection profoundly alters intrahepatic chemokine but not inflammatory cytokine profiles in HCVinfected subjects. 2014, 9(2):e86964. 
19.Jia M, Luo H, Ma Y, Wang N, Smith K, Mei J, Lu R, Lu J, Fu L, Zhang QJJJoAIDS: The HIV epidemic in Yunnan province, China, 1989-2007. 2010, 53:S34-S40.

20.Duan S, Jin Z, Liu X, Yang Y, Ye R, Tang R, Gao M, Ding Y, He NJBo: Tobacco and alcohol use among drug users receiving methadone maintenance treatment: a cross-sectional study in a rural prefecture of Yunnan Province, Southwest China. 2017, 7(3):e014643.

21.Ding Y, Duan S, Ye R, Yang Y, Yao S, Wang J, Cao D, Liu X, Lu L, Jia MJJovh: More improvement than progression of liver fibrosis following antiretroviral therapy in a longitudinal cohort of HIV-infected patients with or without HBV and HCV co-infections. 2017, 24(5):412-420.

22.Tada T, Kumada T, Toyoda H, Kiriyama S, Tanikawa M, Hisanaga Y, Kanamori A, Kitabatake S, Yama T, Tanaka J: Long-term prognosis of patients with chronic hepatitis $C$ who did not receive interferon-based therapy: causes of death and analysis based on the FIB-4 index. Journal of gastroenterology 2016, 51(4):380-389.

23.Sterling RK, Lissen E, Clumeck N, Sola R, Correa MC, Montaner J, M SS, Torriani FJ, Dieterich DT, Thomas DL et al: Development of a simple noninvasive index to predict significant fibrosis in patients with HIV/HCV coinfection. Hepatology (Baltimore, Md) 2006, 43(6):1317-1325.

24.Vallet-Pichard A, Mallet V, Nalpas B, Verkarre V, Nalpas A, Dhalluin-Venier V, Fontaine H, Pol S: FIB-4: an inexpensive and accurate marker of fibrosis in HCV infection. comparison with liver biopsy and fibrotest. Hepatology (Baltimore, Md) 2007, 46(1):32-36.

25.Zhang T, Tully DC, Zhou S, He NJPo: Characteristics of HCV co-infection among HIV infected individuals from an area with high risk of blood-borne infections in central China. 2014, 9(4):e94219.

26.Armstrong RA: When to use the Bonferroni correction. Ophthalmic \& physiological optics: the journal of the British College of Ophthalmic Opticians (Optometrists) 2014, 34(5):502-508.

27.Down C, Mehta N, Marks K: The Risk of Cardiovascular Disease, Diabetes, Liver-Related Outcomes, and Death Over 10 Years in HIV/HCV-Coinfected Patients With and Without Steatosis. AIDS research and human retroviruses 2016, 32(9):868-871.

28.Durier N, Yunihastuti E, Ruxrungtham K, Kinh NV, Kamarulzaman A, Boettiger D, Widhani A, Avihingsanon A, Huy BV, Syed Omar SF et al: Chronic hepatitis $C$ infection and liver disease in HIVcoinfected patients in Asia. Journal of viral hepatitis 2017, 24(3):187-196.

29.Merli M, Galli L, Castagna A, Salpietro S, Gianotti N, Messina E, Poli A, Morsica G, Bagaglio S, Cernuschi $M$ et al: Diagnostic accuracy of APRI, FIB-4 and Forns for the detection of liver cirrhosis in HIV/HCV-coinfected patients. The new microbiologica 2016, 39(2):110-113.

30.Pembroke T, Deschenes M, Lebouche B, Benmassaoud A, Sewitch M, Ghali P, Wong P, Halme A, VuilleLessard E, Pexos C et al: Hepatic steatosis progresses faster in HIV mono-infected than HIV/HCV co- 
infected patients and is associated with liver fibrosis. Journal of hepatology 2017, 67(4):801-808.

31.Beudeker BJB, van Oord GW, Arends JE, Schulze Zur Wiesch J, van der Heide MS, de Knegt RJ, Verbon A, Boonstra A, Claassen MAA: Mucosal-associated invariant T-cell frequency and function in blood and liver of HCV mono- and HCV/HIV co-infected patients with advanced fibrosis. Liver international: official journal of the International Association for the Study of the Liver 2018, 38(3):458-468.

32.Chen ZW, Li Z, Wang QH, Wu XL, Li H, Ren H, Hu P: Large Disparity between Prevalence and Treatment Rates for Hepatitis C in Western China. Journal of clinical and translational hepatology 2018, 6(4):385390.

33.Shmagel KV, Saidakova EV, Shmagel NG, Korolevskaya LB, Chereshnev VA, Robinson J, Grivel JC, Douek DC, Margolis L, Anthony DD et al: Systemic inflammation and liver damage in HIV/hepatitis C virus coinfection. HIV medicine 2016, 17(8):581-589.

34.Funderburg NT, Andrade A, Chan ES, Rosenkranz SL, Lu D, Clagett B, Pilch-Cooper HA, Rodriguez B, Feinberg J, Daar E et al: Dynamics of immune reconstitution and activation markers in HIV+ treatmentnaive patients treated with raltegravir, tenofovir disoproxil fumarate and emtricitabine. PloS one 2013, 8(12):e83514.

35.Sacchi P, Cima S, Zuccaro V, Columpsi P, Sarda C, Mariani M, Puoti M, Bruno R: Understanding the Mechanisms of Fibrogenesis in HIV/HCV-Coinfected Patients: Implications for Clinical Practice. AIDS reviews 2015, 17(3):159-170.

36.Shmagel KV, Saidakova EV: Systemic inflammation and liver damage in HIV/hepatitis C virus coinfection. 2016, 17(8):581-589.

37.Dirchwolf M, Ruf AE: Role of systemic inflammation in cirrhosis: From pathogenesis to prognosis. World journal of hepatology 2015, 7(16):1974-1981.

38.Xu R, Zhang Z, Wang FS: Liver fibrosis: mechanisms of immune-mediated liver injury. Cellular \& molecular immunology 2012, 9(4):296-301.

39.McGaha TL, Bona CA: Role of profibrogenic cytokines secreted by $T$ cells in fibrotic processes in scleroderma. Autoimmunity reviews 2002, 1(3):174-181.

40.Balagopal A, Philp FH, Astemborski J, Block TM, Mehta A, Long R, Kirk GD, Mehta SH, Cox AL, Thomas $\mathrm{DL}$ et al: Human immunodeficiency virus-related microbial translocation and progression of hepatitis $C$. Gastroenterology 2008, 135(1):226-233.

41.de Lalla C, Galli G, Aldrighetti L, Romeo R, Mariani M, Monno A, Nuti S, Colombo M, Callea F, Porcelli SA et al: Production of profibrotic cytokines by invariant NKT cells characterizes cirrhosis progression in chronic viral hepatitis. Journal of immunology (Baltimore, Md: 1950) 2004, 173(2):1417-1425. 
42. Heymann F, Hammerich L, Storch D, Bartneck M, Huss S, Russeler V, Gassler N, Lira SA, Luedde T, Trautwein $\mathrm{C}$ et al: Hepatic macrophage migration and differentiation critical for liver fibrosis is mediated by the chemokine receptor C-C motif chemokine receptor 8 in mice. Hepatology (Baltimore, Md) 2012, 55(3):898-909.

43.Graham CS, Wells A, Liu T, Sherman KE, Peters M, Chung RT, Bhan AK, Andersen J, Koziel MJ: Antigenspecific immune responses and liver histology in HIV and hepatitis C coinfection. AIDS (London, England) 2005, 19(8):767-773.

44.Knight B, Lim R, Yeoh GC, Olynyk JK: Interferon-gamma exacerbates liver damage, the hepatic progenitor cell response and fibrosis in a mouse model of chronic liver injury. Journal of hepatology 2007, 47(6):826-833.

45.Keating SM, Dodge JL, Norris PJ, Heitman J, Gange SJ, French AL, Glesby MJ, Edlin BR, Latham PS, Villacres MC et al: The effect of HIV infection and HCV viremia on inflammatory mediators and hepatic injury-The Women's Interagency HIV Study. PloS one 2017, 12(9):e0181004.

46.Meng F, Wang K, Aoyama T, Grivennikov SI, Paik Y, Scholten D, Cong M, Iwaisako K, Liu X, Zhang M et al: Interleukin-17 signaling in inflammatory, Kupffer cells, and hepatic stellate cells exacerbates liver fibrosis in mice. Gastroenterology 2012, 143(3):765-776.e763.

47.Dirchwolf M, Podhorzer A, Marino M, Shulman C, Cartier M, Zunino M, Paz S, Munoz A, Bocassi A, Gimenez J et al: Immune dysfunction in cirrhosis: Distinct cytokines phenotypes according to cirrhosis severity. Cytokine 2016, 77:14-25.

48.Cheever AW, Williams ME, Wynn TA, Finkelman FD, Seder RA, Cox TM, Hieny S, Caspar P, Sher A: Anti$I L-4$ treatment of Schistosoma mansoni-infected mice inhibits development of $T$ cells and non- $B, n o n-T$ cells expressing Th2 cytokines while decreasing egg-induced hepatic fibrosis. Journal of immunology (Baltimore, Md: 1950) 1994, 153(2):753-759.

49.Kaspar MB, Sterling RK: Mechanisms of liver disease in patients infected with HIV. BMJ open gastroenterology 2017, 4(1):e000166.

50.Zeremski M, Petrovic LM, Chiriboga L, Brown QB, Yee HT, Kinkhabwala M, Jacobson IM, Dimova R, Markatou M, Talal AH: Intrahepatic levels of CXCR3-associated chemokines correlate with liver inflammation and fibrosis in chronic hepatitis C. Hepatology (Baltimore, Md) 2008, 48(5):1440-1450.

51.Tazi KA, Quioc JJ, Abdel-Razek W, Tellier Z, Guichard C, Ogier-Denis E, Lebrec D, Moreau R: Protein array technology to investigate cytokine production by monocytes from patients with advanced alcoholic cirrhosis: An ex vivo pilot study. Hepatology research: the official journal of the Japan Society of Hepatology 2009, 39(7):706-715. 
52.Medrano LM, Garcia-Broncano P, Berenguer J, Gonzalez-Garcia J, Jimenez-Sousa MA, Guardiola JM, Crespo M, Quereda C, Sanz J, Canorea I et al: Elevated liver stiffness is linked to increased biomarkers of inflammation and immune activation in HIV/hepatitis C virus-coinfected patients. AIDS (London, England) 2018, 32(9):1095-1105.

53. Harvey CE, Post JJ, Palladinetti P, Freeman AJ, Ffrench RA, Kumar RK, Marinos G, Lloyd AR: Expression of the chemokine IP-10 (CXCL10) by hepatocytes in chronic hepatitis C virus infection correlates with histological severity and lobular inflammation. Journal of leukocyte biology 2003, 74(3):360-369.

54.Berenguer J, Fernandez-Rodriguez A, Jimenez-Sousa MA, Cosin J, Zarate P, Micheloud D, Lopez JC, Miralles P, Catalan P, Resino S: High plasma CXCL10 levels are associated with HCV-genotype 1, and higher insulin resistance, fibrosis, and HIV viral load in HIV/HCV coinfected patients. Cytokine 2012, 57(1):25-29.

55.Sandler NG, Koh C, Roque A, Eccleston JL, Siegel RB, Demino M, Kleiner DE, Deeks SG, Liang TJ, Heller TJG: Host response to translocated microbial products predicts outcomes of patients with HBV or HCV infection. 2011, 141(4):1220-1230. e1223.

56.Balagopal A, Gama L, Franco V, Russell JN, Quinn J, Higgins Y, Smeaton LM, Clements JE, Thomas DL, Gupta AJPO: Detection of microbial translocation in HIV and SIV infection using the Limulus amebocyte lysate assay is masked by serum and plasma. 2012, 7(8):e41258.

57.French AL, Evans CT, Agniel DM, Cohen MH, Peters M, Landay AL, Desai SNJTJoid: Microbial translocation and liver disease progression in women coinfected with HIV and hepatitis C virus. 2013, 208(4):679-689.

58. Hutchinson SJ, Bird SM, Goldberg DJ: Influence of alcohol on the progression of hepatitis C virus infection: a meta-analysis. Clinical gastroenterology and hepatology: the official clinical practice journal of the American Gastroenterological Association 2005, 3(11):1150-1159.

\section{Tables}

Table 1. Demographic and clinical characteristics of HIV/HCV co-infected participants 


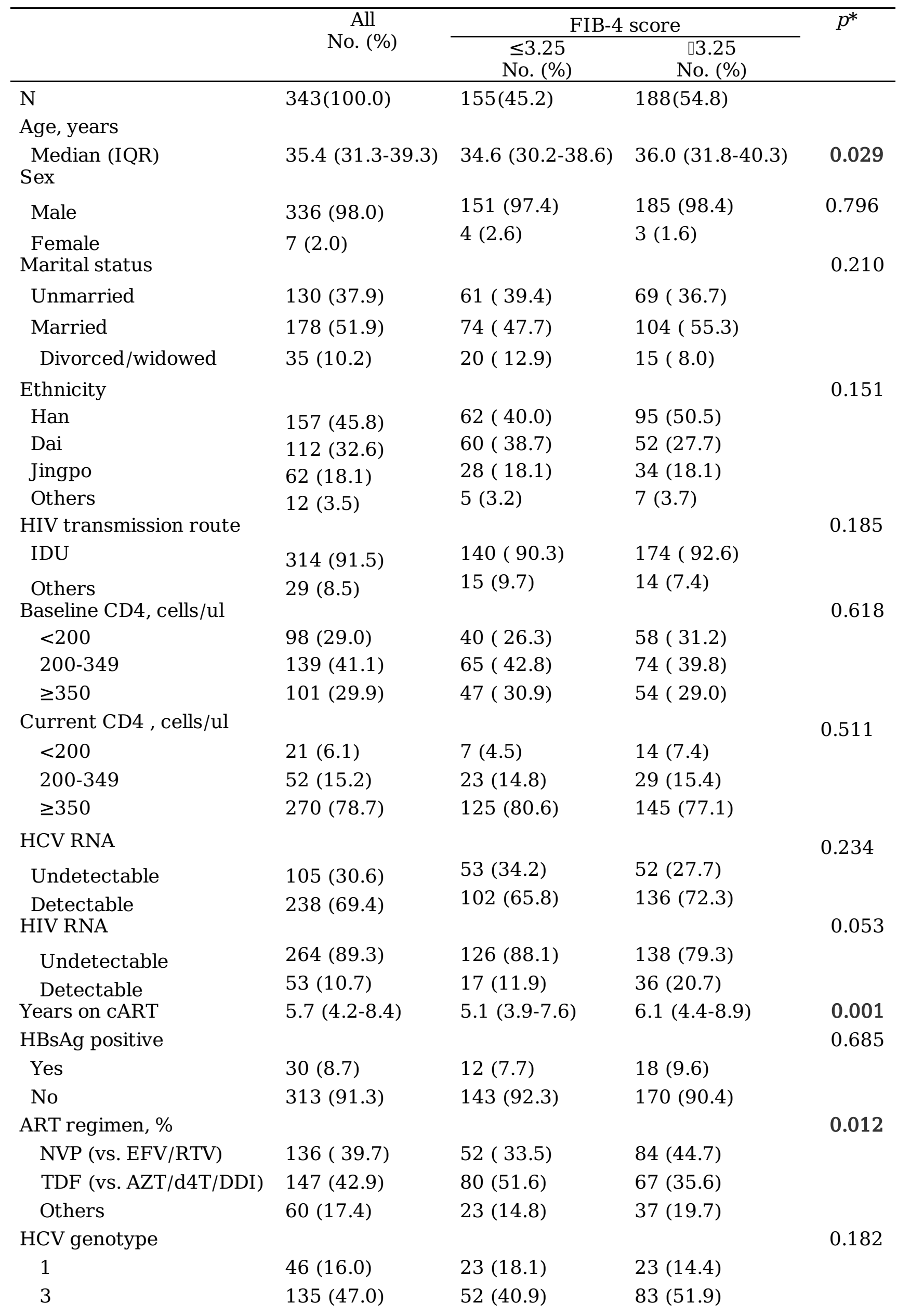


aIDU, injection drug use;

${ }^{b}$ Chi-square tests or Mann-Whitney U test were performed wherever appropriate;

${ }^{\mathrm{c} B o l d}$ signifies statistical significance as $\mathrm{p}<0.05$

Table 2. Plasma level of inflammatory cytokines according to current FIB-4 score 


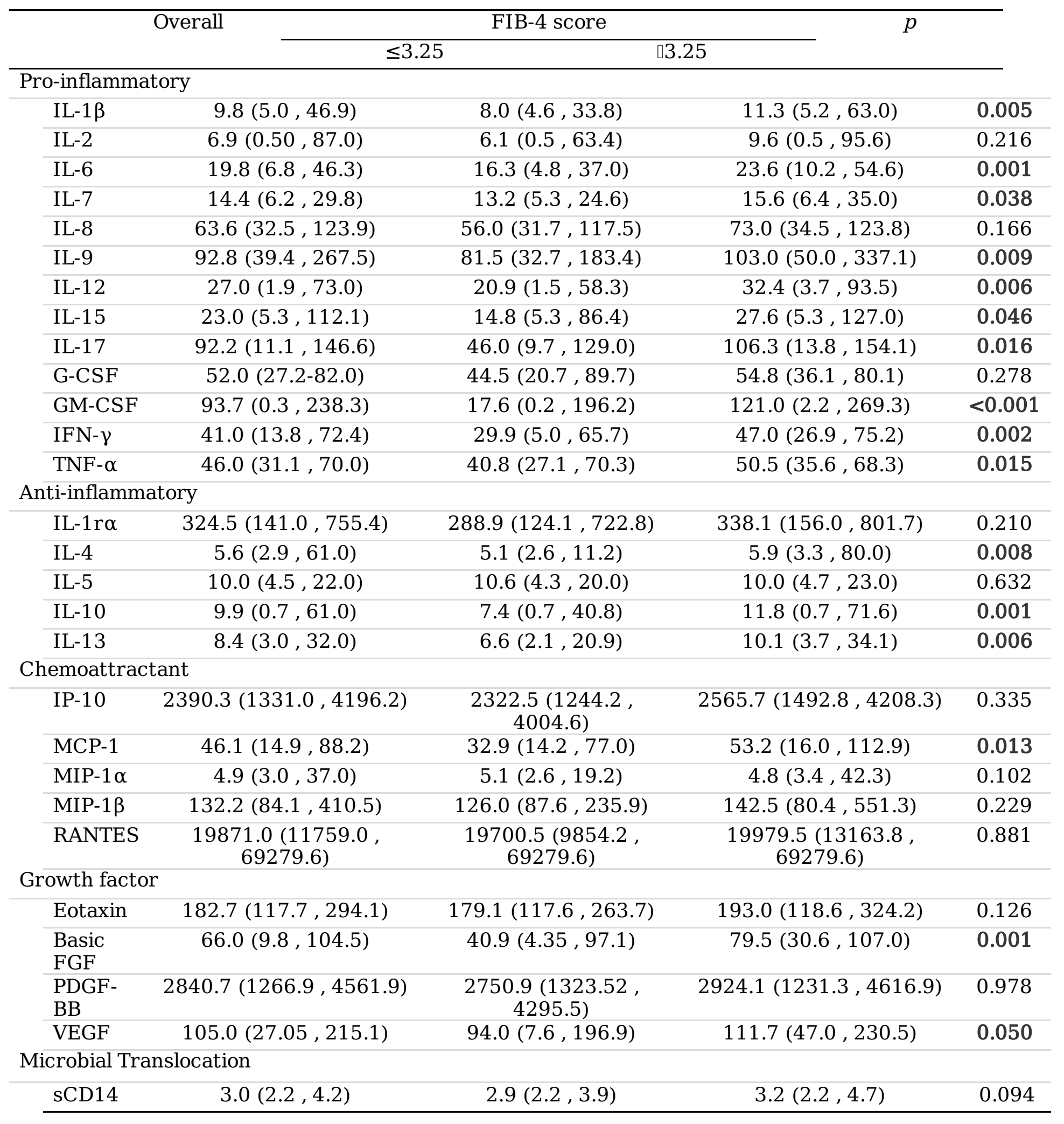

data was presented as median and interquartile range (IQR), with $\mu \mathrm{g} / \mathrm{mL}$ as the unit for sCD14 and $\mathrm{pg} / \mathrm{mL}$ for all other cytokines.

\section{Figures}


FIB4 $\square>3.25 \square \leq 3.25$

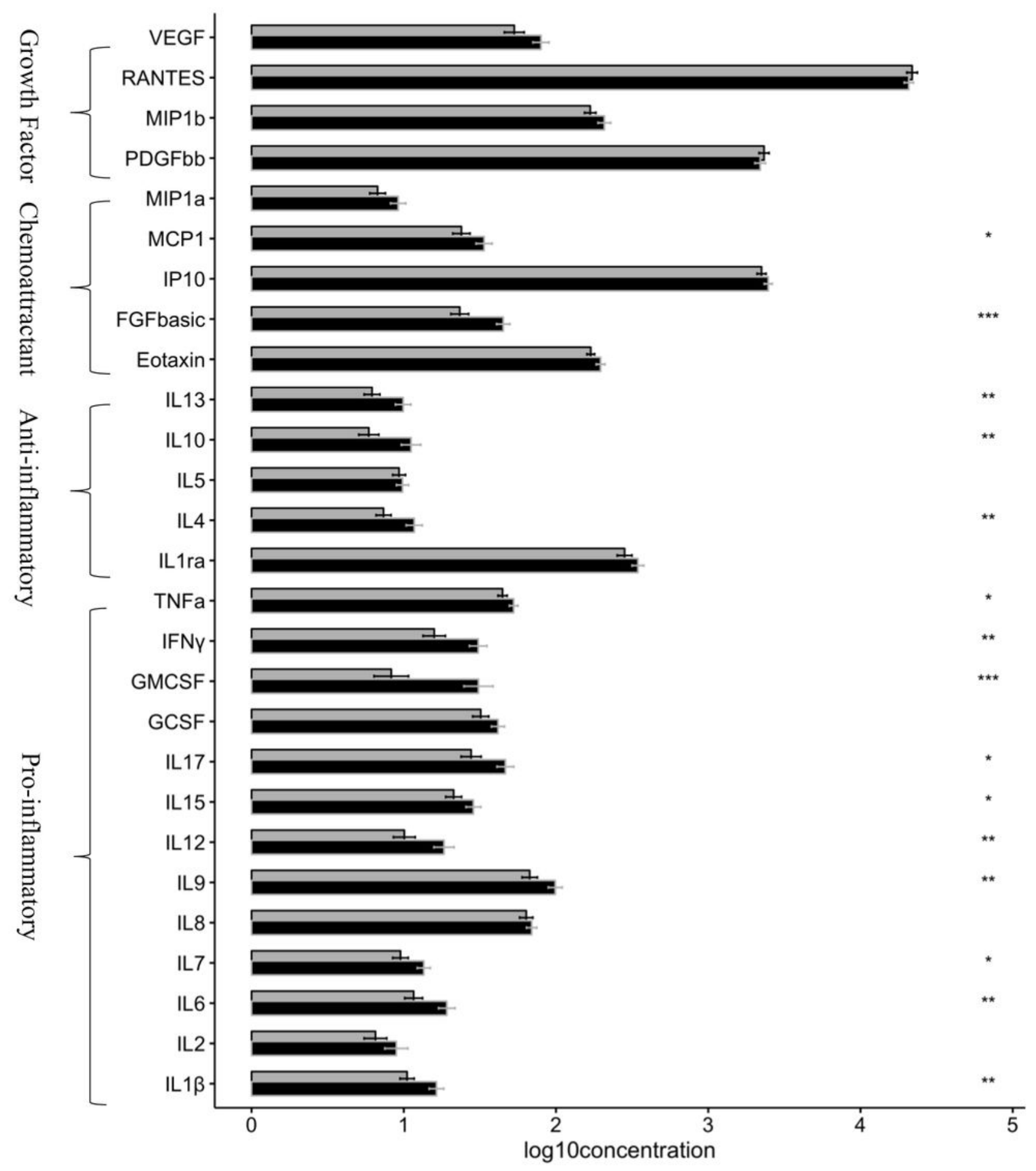

Figure 1

Comparison of log10 transformed plasma concentration of tested biomarkers between participants with different FIB-4 score. ${ }^{\star} p<0.05,{ }^{*} p<0.01$, and ${ }^{\star \star *} p<0.001$. All the $p$-values were calculated by MannWhitney tests. 


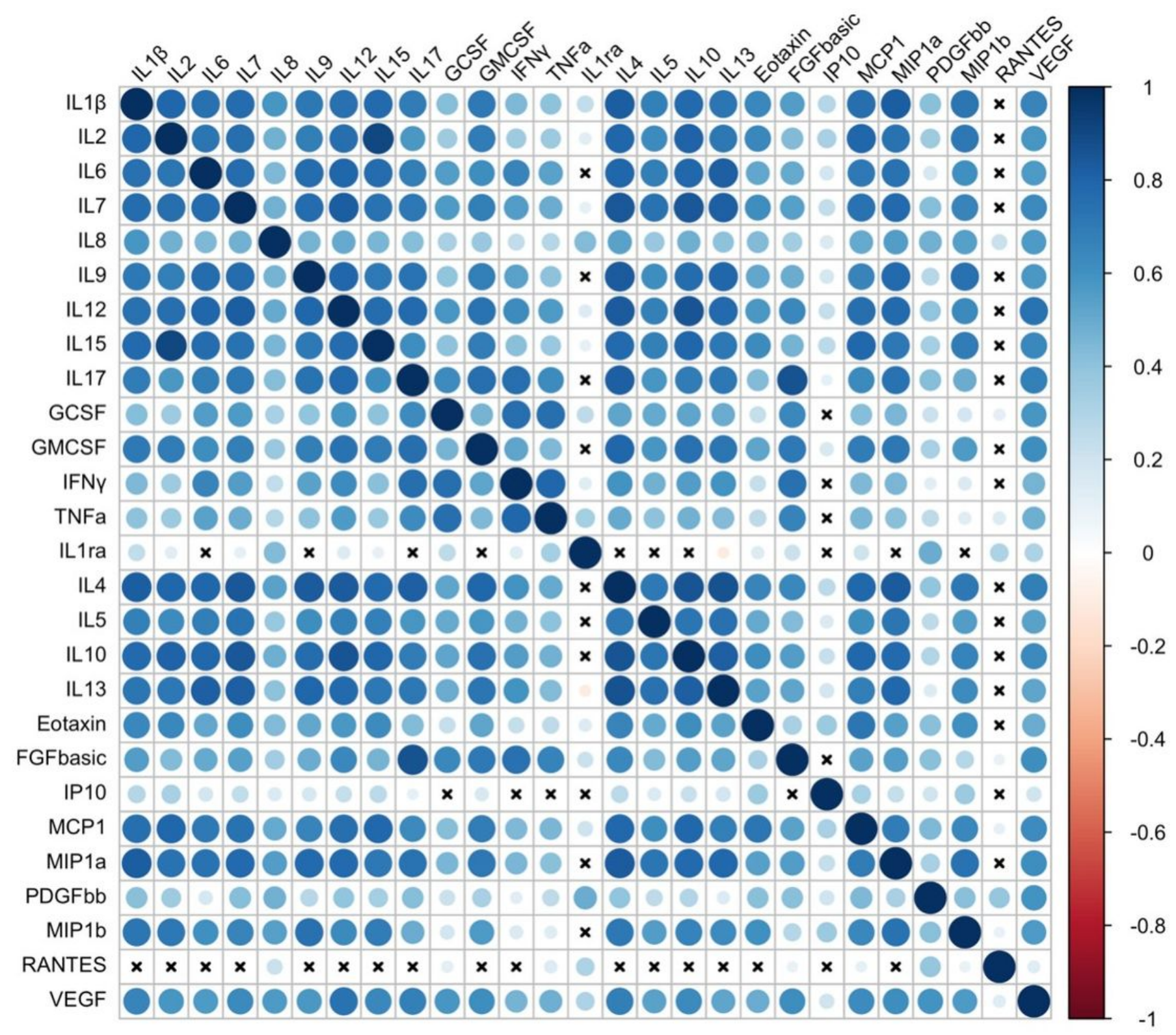

Figure 2

Spearman correlations between plasma inflammatory biomarkers. " $x$ " represents $p \geq 0.05$. Blue and red indicate that the two variables were positively and negatively correlated, respectively. The darker the color (the larger the circle), the greater the correlation of the variables. 


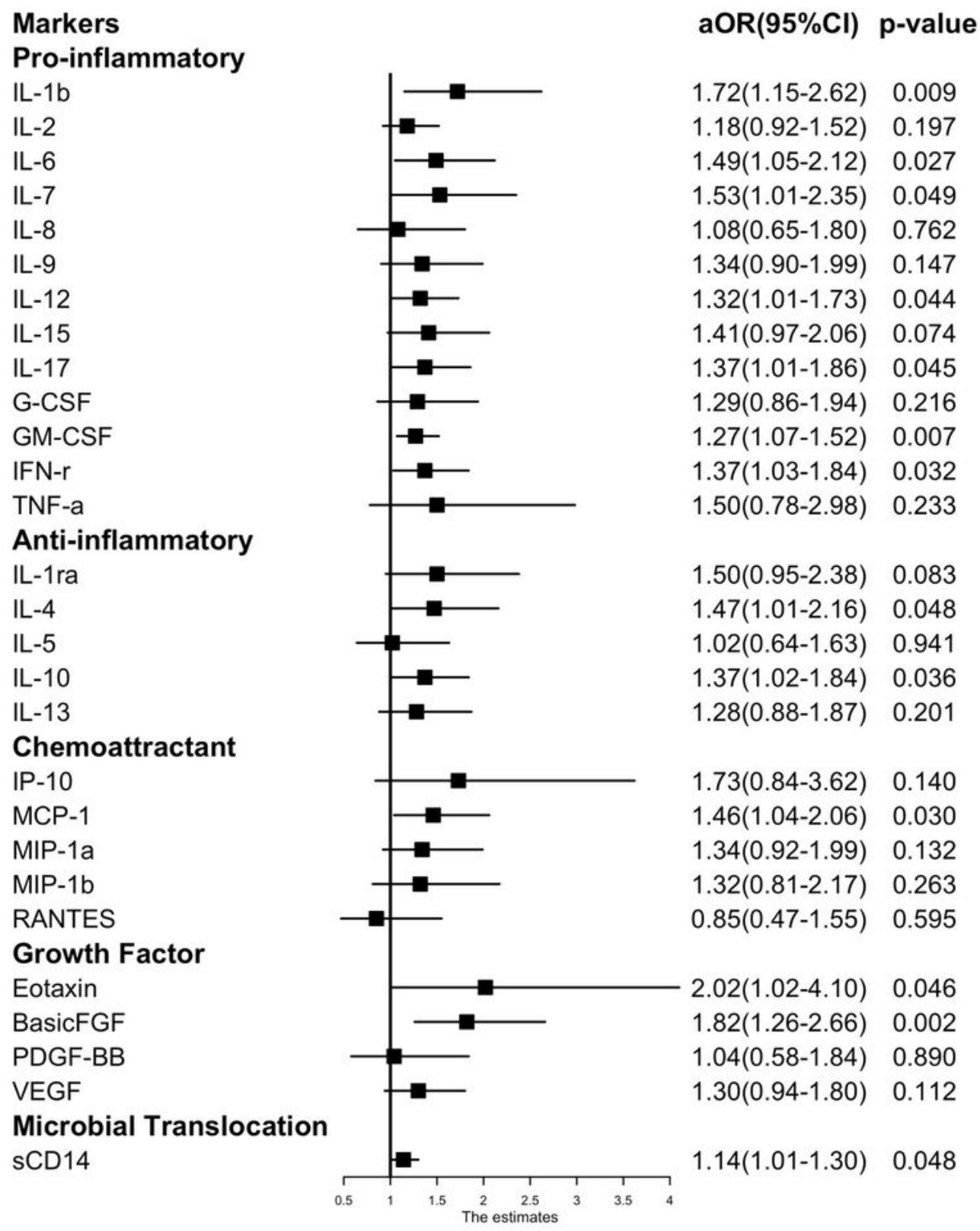

\section{Figure 3}

adjusted Odds ratios and $95 \%$ confidence intervals for the associations between plasma cytokine levels (log10 transformed) and serve liver fibrosis among HIV/HCV co-infected patients. Each variable was assessed in separate multivariable logistic regression adjusting for age, sex, ethnicity, current HIV RNA, current HCV RNA, current CD4 count, years on CART, and ART regimen. 


\section{Supplementary Files}

This is a list of supplementary files associated with this preprint. Click to download.

- supplementary.docx 